\title{
THE RELEASE OF ALARM AND ATTACK BEHAVIOR IN SOME NEW WORLD ARMY ANTS
}

\author{
By William L. Brown, Jr. \\ Museum of Comparative Zoology \\ Harvard University
}

The effect of odoriferous mandibular gland secretions (pheromones) as releasers of alarm behavior in Pogonomyrmex and some other ants has recently been investigated by Wilson ( 1959 and pers. commun.). It is not known how widely among the subfamilies of ants that the same or an homologous effect may occur, but Wilson, myself and others have been able to detect various pungent odors originating from the heads of worker ants in the subfamilies Myrmicinae (Pogonomyrmex, Solenopsis), Ponerinae (Paraponera, Mesoponera, Leptogenys), and Formicinae (Acanthomyops, Lasius). That these substances so often originate in the head is suggestive of a similar source and behavioral function, but in most cases experimental evidence of their function is lacking."

A recent study trip to the Smithsonian Institution's Canal Zone Biological Area station on Barro Colorado Island ${ }^{2}$ afforded a chance to study alarm behavior in the New World army ants (Dorylinae, tribe Ecitonini). Since the trip had other primary purposes, the army ant observations were rather limited, and some obvious tests that suggest themselves, such as trials of interspecific reactions, were not carried out. The results obtained, however, do indicate that the detailed study of army ant pheromones would be a rewarding one. Schneirla (I956 and earlier papers) has made intensive studies of the army ants of Barro Colorado, and has outlined many aspects of their biology, including the remarkably regular nomadic habits of the Eciton species. The first species I studied, E. hamatum (Fabricius), was also the one given the most attention by Schneirla and his colleagues; it is the most conspicuous army ant on the island.

A trunk trail of $E$. hamatum was found at midday running along the edge of a buttress root of a forest tree. The two-way stream of ants varied from two to six individuals in width, and the incoming ants bore much booty, mostly the pupae of the fungus-growing ant,

\footnotetext{
${ }^{1}$ I have found that ants of the Australian formicine genus Calomyrmex produce a bright yellow or scarlet droplet at the base of each mandible when roughly disturbed, but it is not known whether this substance is odoriferous.

${ }^{2}$ The trip was supported by a grant from the Milton Fund of Harvard University.
} 
Atta. A faint "meaty" odor was noticed as I brought my face close to the column; this odor increased markedly when I agitated a part of the column with my machete, and the ants savagely attacked the blade, biting and attempting to sting. (The meatlike odor of some New World army ants has been well known for years.) Selecting one of the large soldiers, with its conspicuous, shiny whitish head and long hooked jaws, I removed the head with forceps and placed it in the column, where it was at once vigorously attacked, bitten and stung by a mass of workers and soldiers that collected around it. The actively struggling body of this same soldier was then placed in the column at another point; the headless body, despite its considerable and highly irregular activity, attracted only momentary antennal play from passing nestmates.

Several more trials like this were made at different points along the column; it was found that soldier heads attracted more attackers than did the much smaller worker heads tried, and also that freshly crushed soldier heads drew a stronger attack than intact ones. To my nose, the strength of the meaty odor in each case appeared to be roughly proportional to the number and activity of the attackers, and I could detect none of this odor from the postcephalic part of the body after the heads had been removed. (Later, in the laboratory, crushed soldier heads of E. hamatum were sniffed by two colleagues and myself, and compared directly with the odor of beef and vegetable bouillon cubes, with the result that all of us found the odors close if not identical.)

Next, small dead twigs were broken in half, and one half rubbed against the crushed head of a worker. Such twigs when placed in the column were subjected to an attack as massive and vigorous as were the crushed heads alone. The untreated half of each twig, serving as a control placed at another point along the column, never received more than fleeting attention from passing soldiers and workers.

Similar results were consistently obtained with another column of E. hamatum found on a subsequent day.

The same kind of investigation was carried out briefly with a column of Nomamyrmex esenbecki (Westwood) found in the forest, utilizing the large and medium-sized workers for the decapitation test (this species lacks large soldiers). To my nose, crushed heads of this species have a different and weaker odor than do E. hamatum workers of the same size, but the alarm and attack reaction they provoked was similar to that of hamatum in intensity.

The test was also made with soldiers from a raiding swarm of 
Labidus praedator (Fr. Smith) found in the Laboratory Clearing on Barro Colorado, and results were again similar to those of the first trials with $E$. hamatum. I could not be sure in this case that I detected any odor at all from the crushed soldier heads of $L$. praedator, but the intensity of attack on these heads indicated that an odor must have been readily detectable by the workers participating.

In summary, the detached heads of workers or soldiers of these army ants from three different genera were attacked by their nestmates when placed in the foraging column, while the remainder of the body evoked little or no response. Objects smeared with the substance from the crushed heads of soldiers drew intense attack, while untreated control objects did not. It seems likely that a pheromone originating in the head, and most likely specifically in the mandibular glands, occurs in the army ants as well as in Pogonomyr$m e x$, and that its function is similar. Apparently, the ants will attack any strange object presented close to the center of concentration of the alarm odor.

The question arises as to how the individuals that are disturbed into producing the alarm odor are not themselves attacked in the normal course of events. Two of the most likely answers that suggest themselves to me are: (I) workers and soldiers, when normally disturbed, give off much lesser amounts of the alarm odor than is produced when a head is severed from the body or crushed, and the attack on severed heads or crushed heads represents a supernormal stimulus; (2) the postcephalic part of the ant's body bears some quality, probably again pheromonal in nature, that neutralizes attack behavior that might otherwise be released in nestmates. The second answer is favored as an hypothesis in the absence of further experiments. If a given soldier were protected by such a neutralizer or "identification pheromone," or by a "nest odor," it would presumably be able to indicate by means of alarm odor release the locality of an intruding disturbance without itself being subject to attack by its nestmates.

I should like to acknowledge helpful suggestions made by Prof. E. O. Wilson during the course of the work and the preparation of the manuscript.

\section{REFERENCES}

SchneIRLA, T. C. 1956. The army ants. In Report of the Smithsonian Institution for 1955, pp. 379-406 (with bibliography).

Wilson, E.O. 1959. (1958). A chemical releaser of alarm and digging behavior in the ant Pogonomyrmex badius Latreille). Psyche. 65: 41-51. 

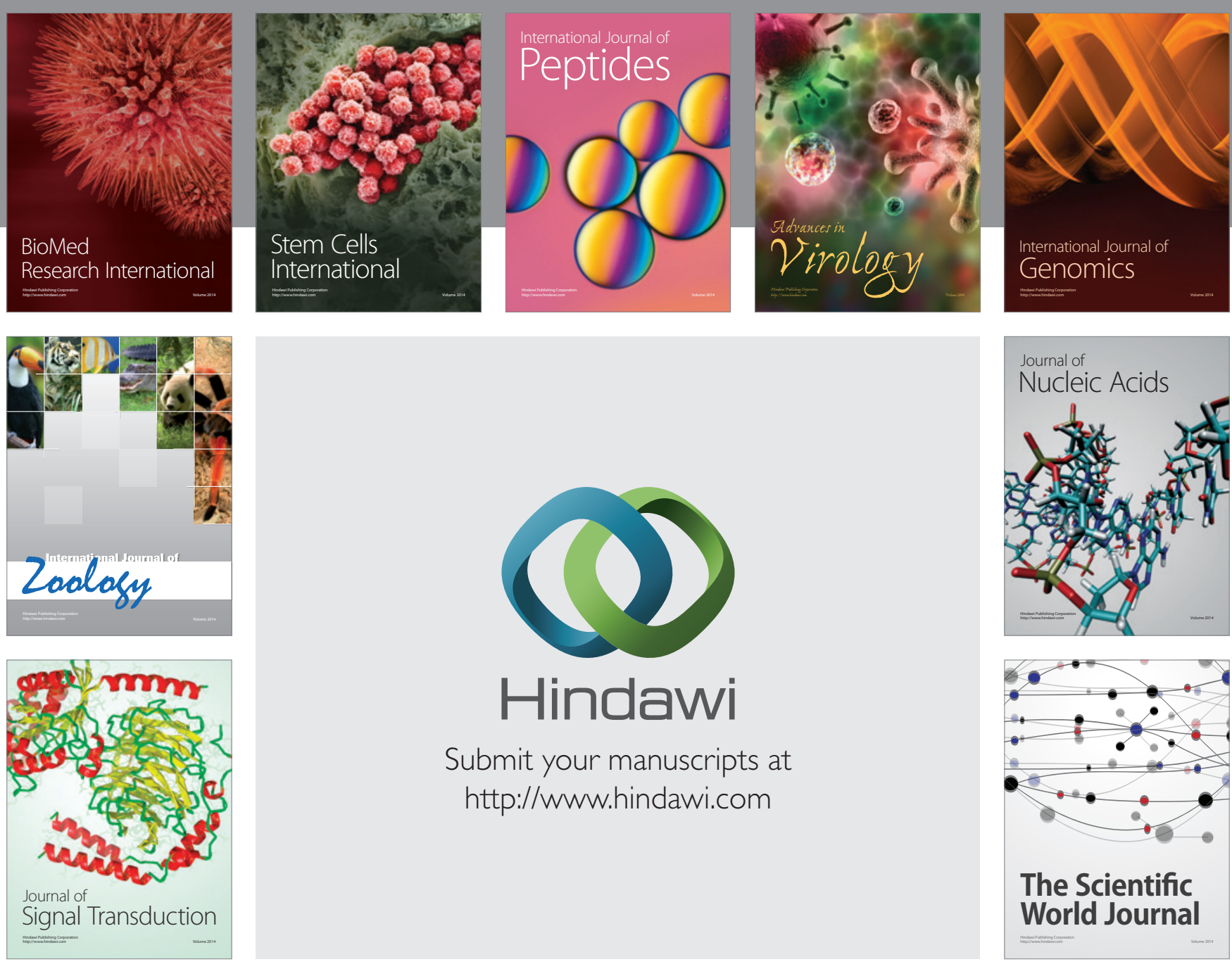

Submit your manuscripts at

http://www.hindawi.com
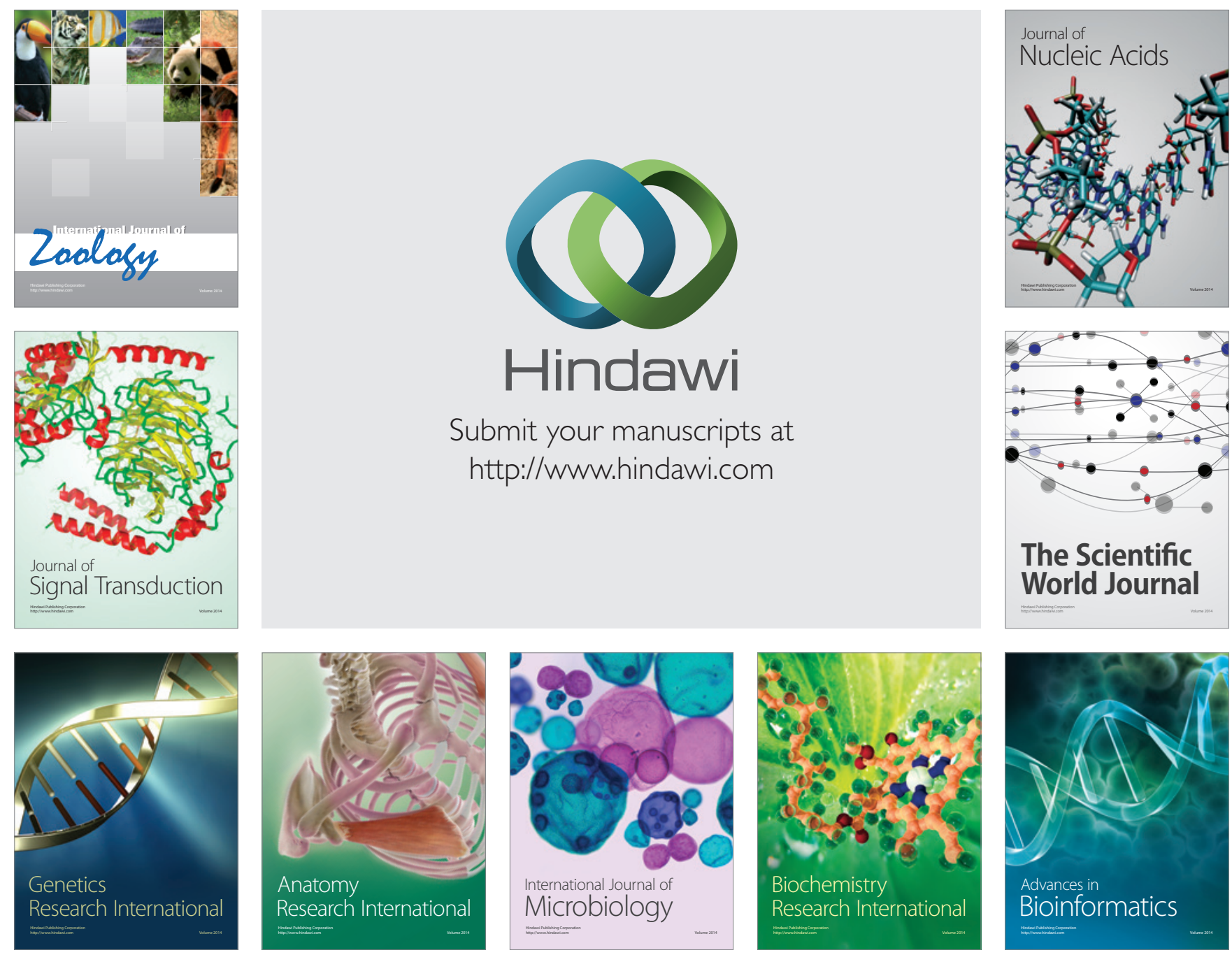

The Scientific World Journal
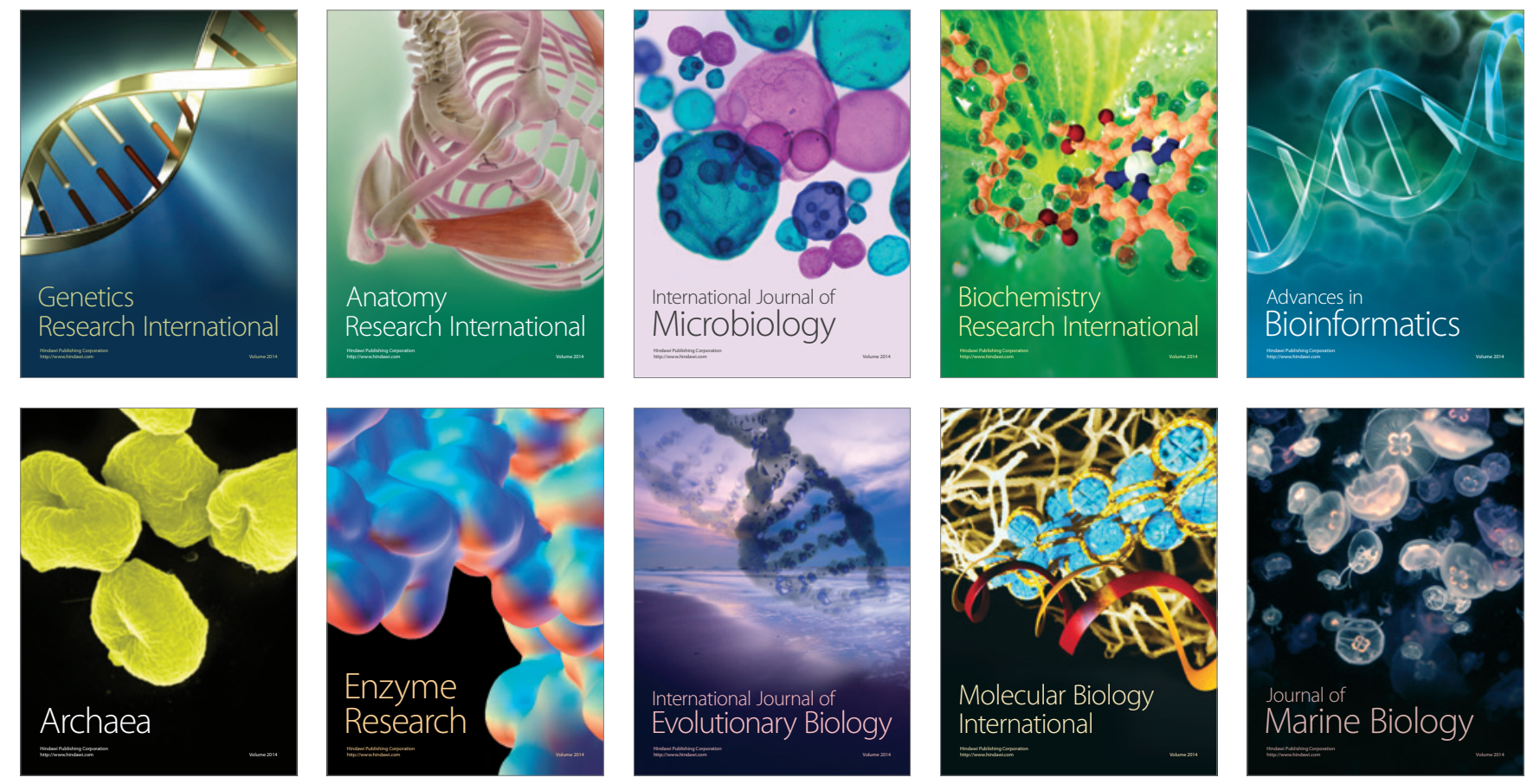